\title{
Spectral Methods in Causal Dynamical Triangulations
}

Giuseppe Clemente*, ${ }^{a b}$ Massimo D’Elia ${ }^{a b}$ and Alessandro Ferraro ${ }^{a b}$

${ }^{a}$ Università di Pisa, Largo B. Pontecorvo 3, I-56127 Pisa, Italy

${ }^{b}$ INFN Sezione di Pisa, Largo B. Pontecorvo 3, I-56127 Pisa, Italy

E-mail: giuseppe.clemente@pi.infn.it, massimo.delia@unipi.it, alessandro.ferraro@i.infn.it

We show recent results of the application of spectral analysis in the setting of the Monte Carlo approach to Quantum Gravity known as Causal Dynamical Triangulations (CDT), discussing the behavior of the lowest lying eigenvalues of the Laplace-Beltrami operator computed on spatial slices. This kind of analysis provides information about running scales of the theory and about the critical behaviour around a possible second order transition in the CDT phase diagram, discussing the implications for the continuum limit.

37th International Symposium on Lattice Field Theory - Lattice2019

16-22 June 2019

Wuhan, China

${ }^{*}$ Speaker. 


\section{Introduction}

Formulating a consistent quantum field theory of gravity has proved to be a difficult task, since the action correctly describing the classical theory, the Einstein-Hilbert action, is nonrenormalizable from a perturbative point of view [1]. However, there is the possibility of a nonperturbative mechanism for renormalization, first envisaged by Weinberg and known as Asymptotic safety scenario [2]: the region of vanishing (bare) couplings in the phase diagram is not necessarily an UV fixed point for the RG flow, but this does not excludes that an UV fixed point actually exists in another region of the phase diagram, not under the reach of perturbative asymptotic series.

The RG flow of the Einstein-Hilbert theory, plus additional terms in the action, can be studied nonperturbatively using Functional Renormalization Group techniques (FRG) [3]. Another nonperturbative approach is numerical, based on Monte Carlo simulations of a lattice-regularized field theory. The approach we discuss in the present report is called Causal Dynamical Triangulations (CDT), where the action used is the Einstein-Hilbert one, discretized in the Regge formalism [4], and with manifolds approximated as piecewise-linear manifolds with appropriate causal conditions. The main goal of the CDT program is to show that, in the phase diagram of the theory, there exists a second order critical point where some definition of correlation length diverges, and that the critical region around such point describes a theory of quantum gravity consistent with our current understanding of the expected semiclassical behavior.

The CDT phase diagram shows a very rich structure with four phase identified as $A, B, C_{d s}$ and $C_{b}$, and two possible second order lines; we refer the reader to $[5,6]$ for more details.

One of the most difficult problems in CDT without matter fields is the definition of local observables that can be associated to correlation lengths, in order to properly investigate the critical properties of the system and the continuum limit.

Some recent advances in this direction came from the introduction of spectral analysis of CDT configurations [7], that is, the analysis of eigenvalues and eigenvectors of the Laplace-Beltrami operator. Here we discuss some of the results obtained, focusing on the analysis of the critical properties of the (seemingly second order) transition line between phase $C_{b}$ and $C_{d S}$, which represents the most promising candidate for taking the continuum limit of a quantum theory of gravity.

\section{Numerical setup and spectral graph methods}

Unlike the similar approach called Euclidean Dynamical Triangulations (EDT) [8, 9, 10, 11, 12], in CDT one enforces a causal condition of global hyperbolicity [13]. This implies that any configuration (simplicial manifold) can be foliated into spatial slices with well defined euclidean time. Due to this condition, and to the absence of singular points in the simplicial manifold, the topology of spatial slices is constant through slices. Here we consider simulations with $S^{3}$ topology of the slices and with periodic conditions in the Euclidean time.

Spatial slices in CDT have only spatial links of uniform length (the lattice spacing); this allows us to faithfully represent them by their dual graph, that is the pair of sets $G=(N, E)$, where the set of nodes $N$ corresponds to spatial tetrahedra, while the set of edges represents the adjacency relations between tetrahedra connected by a facet. The graph analogue of the Laplace-Beltrami 
operator is called the Laplace matrix, which is sparse, linear and symmetric, and acts on the space of real-valued functions on $N\left(\{f: N \rightarrow \mathbb{R}\} \cong \mathbb{R}^{|N|}\right)$; see reference [7] for details.

The updating algorithm used for sampling the path integral is Metropolis-Hastings one, consisting of a set of local moves (see Ref. [5, 6] for more details). In all simulations we used $N_{t}=80$ spatial slices, keeping the total spatial volume $V_{S, t o t}$ fluctuating around a fixed target volume by tuning one of the bare parameters $\left(k_{4}\right)$.

In the following section we introduce some idea which we used to analyze and interpret numerical results.

\subsection{Useful concepts from spectral analysis}

The most interesting spectral quantities, describing the spatial geometries at large scales, are the eigenvalues in the lower spectrum, in particular the lowest (non-zero) one $\lambda_{1}$, which is called spectral gap or usually referred to as algebraic connectivity, since it quantifies the degree of connectedness of the manifold or graph (see ref. [7]). Notice that the LB operator is the one which enters the diffusion equation (or the wave equation), therefore, one can also interpret its eigenvalues as the diffusion rates (or the wavenumber squared) of their associated eigenmodes: a large value of the spectral gap means a fast diffusion (or small wavelength) for the slower mode, which, in turn, means an overconnected geometry, where all space is collapsed into a small region (the diameter is observed to grow logarithmically with the volume). Another quantity of interest is $n(\lambda)$, which counts how many eigenvalues fall below $\lambda$, which, for smooth manifolds, has an useful asymptotic behaviour called Weyl's law [14]:

$$
n(\lambda) \sim \frac{\omega_{d}}{(2 \pi)^{d}} V \lambda^{d / 2}
$$

where $V$ and $d$ are respectively the volume and the local dimension of the manifold, while $\omega_{d}$ is the volume of a $d$-dimensional ball of unit radius. Inspired by Eq. (2.1), we defined a new function $d_{E F F}(\lambda)$, whose role is the one of an effective dimension of the manifold as a function of the length-scale (dictated by $\lambda$ ):

$$
d_{E F F}(\lambda) \equiv 2 \frac{d \log (n / V)}{d \log \lambda}
$$

\section{Numerical Results}

We begin by considering the spectra of the Laplace matrix for spatial slices of configurations deep into the $A, B$ and $C_{d S}$.

Eqs. (2.1) and (2.2) suggest the possibility of an universal scaling of $n / V_{S}$ as a function of $\lambda$ : indeed we observe a remarkable collapse in the scatter plots $\lambda$ vs $n / V_{S}$, independent of the slice (spatial) volume $V_{S}$, but dependent, in general, on the position in the phase diagram. Figure 1 shows the averages of $\lambda$ in bins of $n / V_{S}$, for the three phases aforementioned; these curves are then used to estimate the running effective dimension $d_{E F F}$ by Eq. (2.2), as shown in Figure 2.

The main distinguishing feature of spectra of slices in phase $B$ (actually its unique spatial slice for each configuration) is the presence of a large spectral gap $\lambda_{1}$, which is observed to be independent on the total volume. As argued in the previous section, this large value is associated to a highlyconnected geometry: indeed, the diverging effective dimension at larger scales (small $n / V_{S}$ from 
Weyl's correspondence) suggests that slices in $B$ phase do not seem similar to manifolds at all. The $C_{d S}$ and $A$ phases, even if differing by the correlation between slices with near slice-times (high for $C_{d S}$, but vanishing for $A$ ), show a similar behavior of the $\lambda$ vs $n / V_{S}$ distributions. In particular, we observe a vanishing spectral gap, with a power-like scaling at the large scales; the running effective dimension observed stays relatively constant in the large to intermediate scale, but here it is fractional, showing a fractal-like behavior of the geometry.

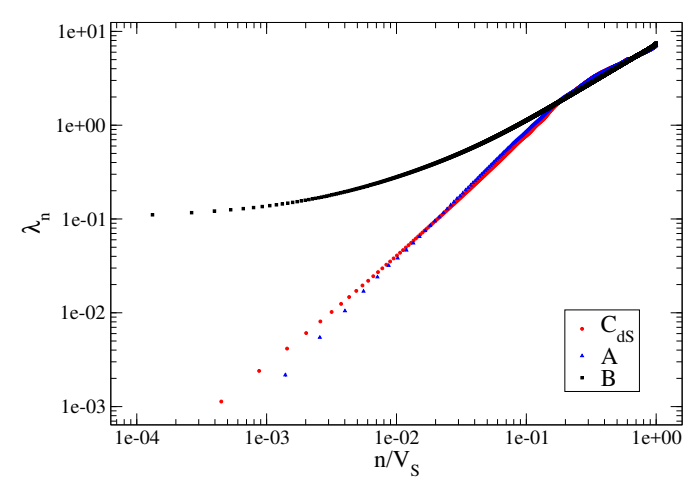

Figure 1: Averages of $\lambda_{n}$ versus $n / V_{S}$ computed in bins of $n / V_{S}$ for slices taken from configurations sampled deep into the $A, B$ and $C_{d S}$ phases: $\left(k_{0}, \Delta\right)=(5,0.6),(2.2,-0.2)$ and $(2.2,0.6)$ respectively. The volume is fixed to $V_{S, t o t}=40 k$ for configurations in $A$ and $C_{d S}$ phase, and to $V_{S, t o t}=8 k$ for configurations in $B$ phase.

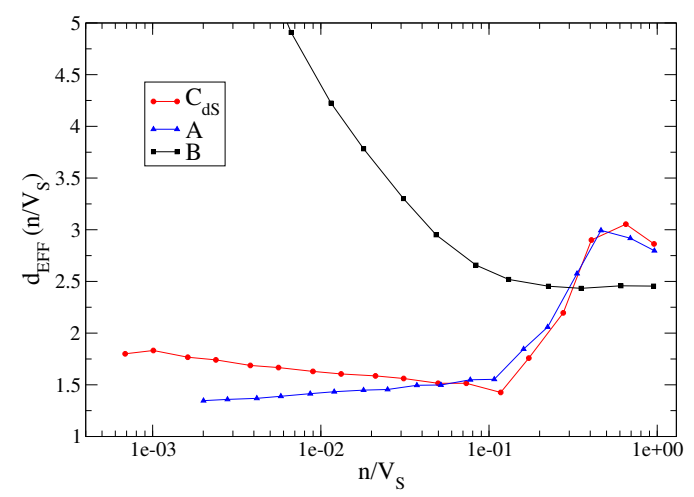

Figure 2: Running effective dimension $d_{E F F}$ (using definition Weyl's law) for the curves shown on the left Figure, obtained by running averages of the logarithmic derivative. The curve associated to the $B$ phase is diverging for $n / V_{S} \rightarrow$ 0 , but we focused on the range $d_{E F F} \in[1,5]$ for readability of the other two curves.

\subsection{Spectral properties of the $C_{b}$ phase}

Configurations in the $C_{b}$ phase have the characteristic feature that their slices have geometric properties which alternate in the slice-time $[15,16]$ so that two distinct classes of slices can be identified. This fact is evident in the low-lying spectra, as can be seen in Figure 3: slices in the bulk of $C_{b}$ phase configurations can be separated in two distinct classes by the value of their spectral gap, changing abruptly for adjacent slices even by two orders of magnitude; in the $C_{d S}$ phase, instead, there is no sharp distinction, apart from a volume-dependent behavior connected to Weyllike scaling. Indeed, the geometric properties of the two classes of slices parallel the properties of the $B$ phase (for the gapped ones) and of the $C_{d S}$ phase (for the non-gapped ones), which brought us to call them respectively $B$-like and $d S$-like. A more general view of the distribution of spectral gaps for different points of the phase diagram (in a fixed $k_{0}=2.2$ line) is given in Figure 4, which shows well the separation of in the two classes for $C_{b}$ slices, and how this separation disappears in going from the $C_{b}$ to the $C_{d S}$ phase. This observation suggests to use the spectral gap of $B$-like slices in the $C_{b}$ phase as an order parameter for the $C_{b}-C_{d S}$ transition, which in literature has been claimed to be second-order by using different methods $[15,16]$. This will be the focus of the next section. 


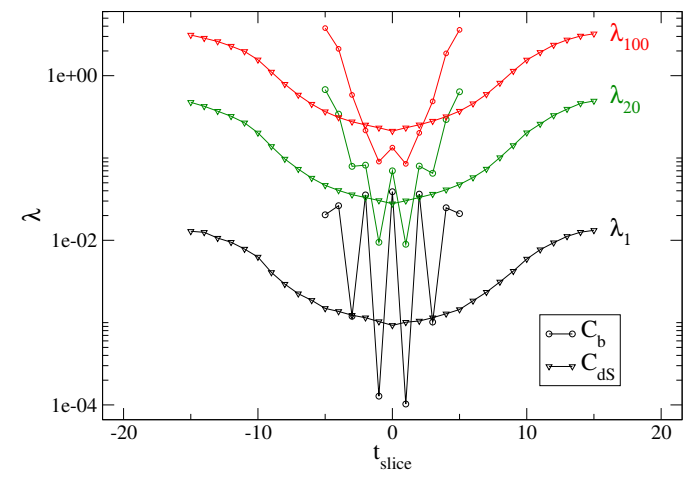

Figure 3: Averages of $\lambda_{1}, \lambda_{20}$ and $\lambda_{100}$ as a function of the slice-time for configurations in $C_{b}$ and $C_{d S}$ phases.

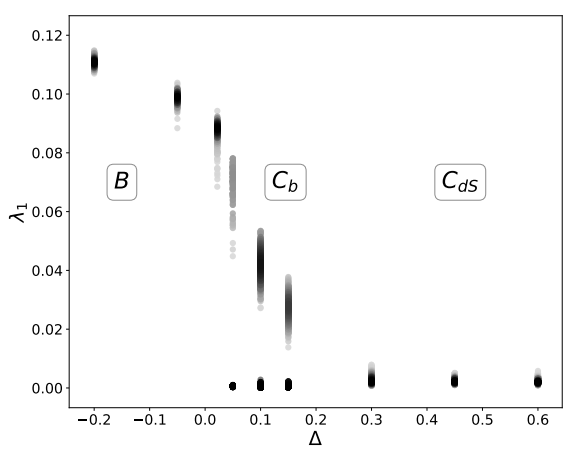

Figure 4: Distributions of $\lambda_{1}$ (vertically) for $k_{0}=2.2$ and variable $\Delta$ for configurations with total spatial volume $V_{S, t o t}=\frac{N_{41}}{2}=40 \mathrm{k}$

\subsection{Spectral study of the critical properties of the $C_{b}-C_{d S}$ transition}

First of all, in order to use the spectral gap of $B$-like slices in $C_{b}$ configurations, we need to study separately the two classes of slices (details can be found in [17]). Figure 5 shows a plot of $\left\langle\lambda_{n}\right\rangle$ vs $1 / V_{S}$ for the $d S$-like class, where the behavior is compatible $\left(\chi^{2} /\right.$ d.o.f. $\left.\simeq 1\right)$ with a vanishing gap in the thermodynamical limit (as expected by Weyl's law). The picture is different for $B$-like slices (Figure 6), where the extrapolations to thermodynamical limit show, not only a non-vanishing spectral gap $\left\langle\lambda_{1}\right\rangle_{\infty}$, but distinct values of the extrapolations for different eigenvalues $\left\langle\lambda_{n}\right\rangle_{\infty}$; this fact will be discussed in the conclusions.

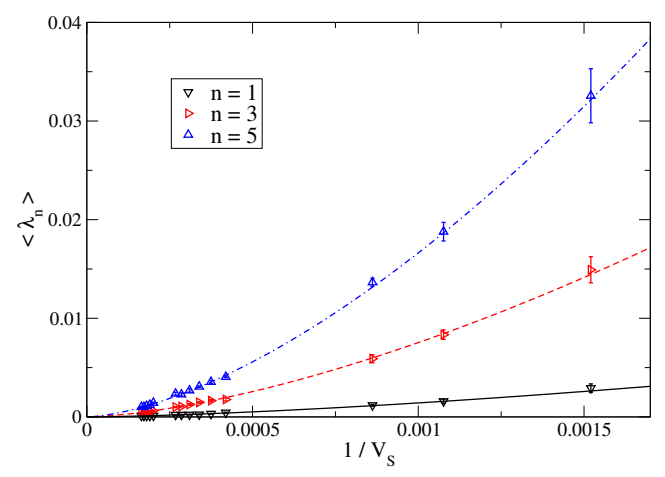

Figure 5: Weyl scaling for some eigenvalue $\left\langle\lambda_{n}\right\rangle(n=1,3,5)$ of $d S$-like slices at $\left(k_{0}, \Delta\right)=$ $(0.75,0.4)$, with vanishing extrapolations in the thermodynamical limit.

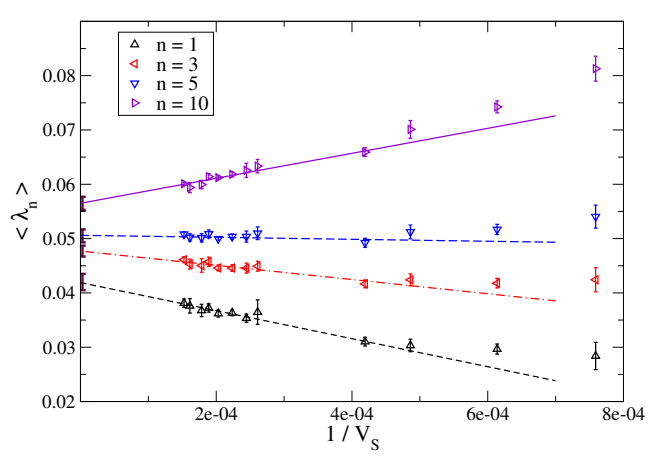

Figure 6: Weyl scaling for some eigenvalue $\left\langle\lambda_{n}\right\rangle(n=1,3,5)$ of $B$-like slices at $\left(k_{0}, \Delta\right)=$ $(0.75,0.4)$, where the thermodynamical limit extrapolations are shown on the vertical axis.

In Figure 7 we report some $\left\langle\lambda_{n}\right\rangle_{\infty}(n=1$ and 5) as a function of $\Delta$ along two lines of simulations at $k_{0}=0.75$ and $k_{0}=1.5$; since, on the immediate vicinity of the critical point, $B$-like and $d S$ like classes mix, it is difficult to separate them and take the thermodynamical limit, therefore, some values of $\Delta$ are excluded from Figure 7 . 


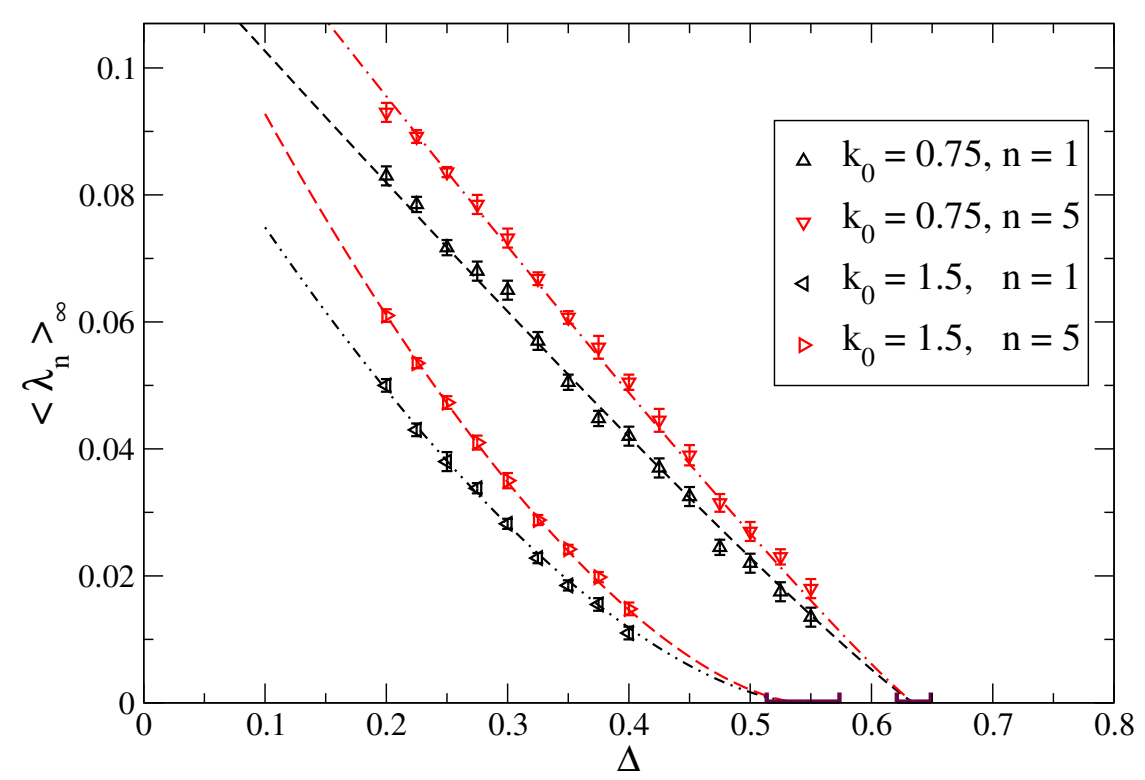

Figure 7: $\left\langle\lambda_{n}\right\rangle_{\infty}$ in $B$-like slices as a function of $\Delta$ for different values of $k_{0}$ and $n$, together with best fits of the scaling equation.

On dimensional grounds, different $\left\langle\lambda_{n}\right\rangle_{\infty}$ are associated to different inverse squared lengths, which however, for the continuum limit to be meaningful, should scale proportionally to each other around a critical point, i.e., with a common critical index. Based on this observation, we tried to fit the data using the scaling formula

$$
\left\langle\lambda_{n}\right\rangle_{\infty}=A_{n}\left(\Delta_{c}-\Delta\right)^{2 v}
$$

where only the $A_{n}$ coefficients depend on $n$. A combined fit, including $n=1,5$, yields $\Delta_{c}=$ $0.635(14), v=0.55(4)$ for $k_{0}=0.75\left(\chi^{2} /\right.$ d.o.f. $\left.=31 / 26\right)$, and $\Delta_{c}=0.544(36), v=0.82(12)$ for $k_{0}=1.50\left(\chi^{2} /\right.$ d.o.f. $\left.=6 / 14\right)$. Similar and consistent results are obtained including different values of $n$, or if the eigenvalues are fitted separately. In principle, our best fits suggest that the index $v$ may change along the transition line; however, a global fit in which $v$ is set equal for both $k_{0}$ works equally well, yielding $v=0.59(4), \Delta_{c}\left(k_{0}=0.75\right)=0.656(15), \Delta_{c}\left(k_{0}=1.5\right)=0.479(10)$ with $\chi^{2} /$ d.o.f. $=47 / 41$.

\section{Conclusions}

We discussed some recent results obtained from the analysis of the spectrum of the LB operator define on spatial slices of CDT configurations [7, 17], focusing in particular on the role of the $C_{b}$ phase and the $C_{b}-C_{d S}$ transition, which represents the most promising candidate for a continuum limit. In particular, we have shown that the two classes of slices in the $C_{b}$ phase have geometric characteristics analogue to the $B$ and $C_{d S}$ phases, with a gapped and non-gapped structure of the spectra respectively.

The thermodynamical limit of the lowest eigenvalues $\left\langle\lambda_{n}\right\rangle_{\infty}$ of $B$-like slices shows the emergence of a hierarchy of distinct characteristic lengths, which could be interpreted as a set of dynamical 
mass scales, connected to the propagation of massless scalar fields in the sampled geometries. Approaching the $C_{d S}$ phase, these mass scales appear to vanish with a common critical index, as expected by a lattice field theory approaching the continuum limit.

\section{References}

[1] M. H. Goroff, A. Sagnotti, The Ultraviolet Behavior of Einstein Gravity, Nucl. Phys. B 266 (1986) 709.

[2] S. Weinberg, General Relativity, an Einstein Centenary Survey, ch.16 Cambridge Univ. Press, 1979.

[3] M. Reuter and F. Saueressig, Quantum Einstein Gravity, New J. Phys. 14 (2012) 055022 [arXiv:1202.2274 [hep-th]].

[4] T. Regge, General Relativity Without Coordinates, Nuovo Cim. 19, 558 (1961).

[5] J. Ambjorn, A. Goerlich, J. Jurkiewicz and R. Loll, Nonperturbative Quantum Gravity, Phys. Rept. 519, 127 (2012) [arXiv:1203.3591 [hep-th]].

[6] R. Loll, Quantum Gravity from Causal Dynamical Triangulations: A Review, [arXiv:1905.08669 [hep-th]].

[7] G. Clemente and M. D'Elia, Spectrum of the Laplace-Beltrami operator and the phase structure of causal dynamical triangulations, Phys. Rev. D 97 (2018) no.12, 124022 doi:10.1103/PhysRevD.97.124022 [arXiv:1804.02294 [hep-th]].

[8] J. Ambjorn and J. Jurkiewicz, Four-dimensional simplicial quantum gravity, Phys. Lett. B 278, 42 (1992).

[9] M. E. Agishtein and A. A. Migdal, Simulations of four-dimensional simplicial quantum gravity, Mod. Phys. Lett. A 7, 1039 (1992).

[10] J. Ambjorn, J. Jurkiewicz and C. F. Kristjansen, Quantum gravity, Nucl. Phys. B 393 (1993) 601 [hep-th/9208032].

[11] T. Rindlisbacher and P. de Forcrand, Euclidean Dynamical Triangulation revisited: is the phase transition really 1st order? (extended version), JHEP 1505 (2015) 138 [arXiv: 1503.03706 [hep-lat]].

[12] J. Laiho, S. Bassler, D. Coumbe, D. Du and J. T. Neelakanta, Lattice Quantum Gravity and Asymptotic Safety, Phys. Rev. D 96 (2017) no.6, 064015 [arXiv:1604.02745 [hep-th]].

[13] E. Minguzzi and M. Sanchez, The Causal hierarchy of spacetimes, EMS Pub.House, 2008, p.299-358 [gr-qc/0609119].

[14] H. Weyl, Über die asymptotische Verteilung der Eigenwerte", Nachr. Konigl. Ges. Wiss. Göttingen, 110-117 (1911).

[15] J. Ambjorn, J. Gizbert-Studnicki, A. Goerlich, J. Jurkiewicz, N. Klitgaard and R. Loll, Characteristics of the new phase in CDT, Eur. Phys. J. C 77 (2017) no.3, 152 [arXiv:1610.05245 [hep-th]].

[16] J. Ambjorn, D. Coumbe, J. Gizbert-Studnicki, A. Goerlich and J. Jurkiewicz, New higher-order transition in causal dynamical triangulations, Phys. Rev. D 95 (2017) no.12, 124029 [arXiv:1704.04373 [hep-lat]].

[17] G. Clemente, M. D'Elia and A. Ferraro, Running scales in causal dynamical triangulations, Phys. Rev. D 99 (2019) no.11, 114506 doi:10.1103/PhysRevD.99.114506 [arXiv: 1903.00430 [hep-th]]. 